\title{
ASYMPTOTIC CYCLES FOR ACTIONS OF LIE GROUPS
}

\author{
SOL SCHWARTZMAN
}

(Communicated by Kailash C. Misra)

\begin{abstract}
Let $M^{k}$ be a compact $C^{\infty}$ manifold and suppose we are given a $C^{\infty}$ action of $\mathbb{R}^{n}$ on $M^{k}$. If $p$ is a quasiregular point for this action and $v$ is an $r$-vector over the Lie algebra of $\mathbb{R}^{n}$, we show how to associate with $p$ and $v$ an element $A_{p}^{v}$ in $H_{r}\left(M^{k} ; \mathbb{R}\right)$. When $n=1$ and $v$ is the usual generator for the Lie algebra of $\mathbb{R}, A_{p}^{v}$ coincides with the asymptotic cycle associated with $p$ by our flow. Just as in the one dimensional case, with any invariant probability measure we can associate an element $A_{\mu}^{v}$ in $H_{r}\left(M^{k} ; \mathbb{R}\right)$.

Several results known in the one dimensional case generalize to our present situation. The results we have stated for actions of $\mathbb{R}^{n}$ are obtained from a discussion of what we can say when we have a smooth action of an arbitrary connected Lie group on $M^{k}$.
\end{abstract}

\section{INTRODUCTION}

Let $M^{k}$ be a compact $C^{\infty}$ manifold and let $G$ be a connected Lie group acting smoothly on $M^{k}$. When $G=\mathbb{R}$, the notion of asymptotic cycle was introduced in [1]. (For a report on what has been done in the literature with this notion, see [4].)

In [2, a case where $G$ could be any Lie group was considered. It was assumed there that all orbits were of the same dimension $r$ and that they were continuously oriented. It was shown how with any finite invariant measure $\mu$ one could associate an element of $H_{r}\left(M^{k} ; \mathbb{R}\right)$, provided one was given what was called a positive quantifier.

In this paper, the action of $G$ on $M^{k}$ will not be subject to any restrictions whatsoever. Given a finite invariant measure, we will nevertheless be able to get an element of $H_{r}\left(M^{k} ; \mathbb{R}\right)$. Instead of our needing a positive quantifier (a concept that would not make sense here), what we need to be given here is an $r$-vector $v$ that is a finite sum of $r$-vectors, each of which is an exterior product of $r$ 1-vectors from the Lie algebra of $G$ that commute with each other. If $G=\mathbb{R}$ and we take as our 1-vector the obvious generator of the one dimensional Lie algebra of $\mathbb{R}$, what we get is called in [1] the $\mu$-asymptotic cycle associated with the invariant measure $\mu$ for our flow.

With this done, in the second part of this paper we will consider the case where $G=\mathbb{R}^{n}$. This case was looked at in [3], but in that paper the only invariant measures that were considered were assumed to be carried on the union of all the orbits of dimension equal to a fixed integer $r$. Given our action of $\mathbb{R}^{n}$ on $M^{k}$, we considered pairs $(P, o)$ where $P$ lay on an $r$-dimensional orbit, and $o$ was an orientation of that orbit. It turned out that we could associate with "almost all" such pairs

Received by the editors February 3, 2011 and, in revised form, September 7, 2011.

2010 Mathematics Subject Classification. Primary 28D15, 54H20. 
(with respect to every one of our invariant measures) an element of $H_{r}\left(M^{k} ; \mathbb{R}\right)$. What "almost all" means here requires a rather involved definition, and the entire discussion in [3] is much more complicated than what we will do here.

For this paper, we will require that we be given some $r$-vector $v$ over the Lie algebra of $\mathbb{R}^{n}$. With any quasi-regular point $p$ in $M^{k}$ we will associate an asymptotic cycle $A_{p}^{v}$. Since it is known that a quasi-regular point $p$ determines an invariant probability measure $\mu_{p}$, the previous discussion tells us immediately how to get an element of $H_{r}\left(M^{k} ; \mathbb{R}\right)$. Just as in the 1-dimensional case, one can arrive at this asymptotic cycle by an appropriate averaging process over the orbit of $p$. Moreover, for any of our invariant measures $\mu, \int A_{p}^{v} d \mu(p)$ equals the element of $H_{r}\left(M^{k} ; \mathbb{R}\right)$ arising from $\mu$ and $v$.

Theorem 1. Suppose that $v$ is a finite sum of $r$-vectors, where each of these $r$ vectors is an exterior product of $r$ commuting 1-vectors from the Lie algebra of $G$. Suppose further that we are given an invariant measure $\mu$. Then for any closed $r$-form $\lambda$, the quantity

$$
\int_{M_{k}} V_{p}^{v}-\lambda(p) d \mu(p)
$$

is unchanged if we substitute for $\lambda$ any other $r$-form determining the same element of $H^{r}\left(M^{k} ; \mathbb{R}\right)$ (here $V_{p}^{v}$ is the $r$-vector over the tangent space at $p$ determined by $v)$.

Once we prove that if $\lambda$ is a bounding $r$-form, then this integral equals zero, it will indeed follow that for $\lambda$ a closed form, this integral depends only on the element of $H^{r}\left(M^{k} ; \mathbb{R}\right)$ determined by $\lambda$. Thus given $\mu$ and $v$, we get a homomorphism of $H^{r}\left(M^{k} ; \mathbb{R}\right)$ into $\mathbb{R}$ and consequently an element of $H_{r}\left(M^{k} ; \mathbb{R}\right)$, which we refer to as the $\mu$-asymptotic cycle associated with $v$ and $\mu$ and designate by $A_{\mu}^{v}$.

Thus to prove Theorem 1, we must prove that if $B$ is a smooth $(r-1)$-form and $\mu$ is a finite invariant measure, then

$$
\int V_{p}-[(d B)(p)] d \mu(p)=0 .
$$

It will be enough to restrict our attention to the case where $v=v_{1} \wedge \ldots \wedge v_{r}$ and the $v_{i}$ commute. We will proceed by induction. Certainly when $r=1$, the result is known to be true. Assume that the result is known for $r \leq n-1$. Then note that

$$
\int D_{V_{r}}\left[\left(V_{1} \wedge \cdots \wedge V_{r-1}\right)-B\right] d \mu=0,
$$

where $D_{V_{r}}$ denotes Lie differentiation with respect to the flow defined by the vector field $V_{r}$, since $\int_{M^{k}}\left[D_{V_{r}}(F)\right](p) d \mu(p)$ equals zero for any smooth function $F$. However,

$$
\begin{aligned}
\left.D_{V_{r}}\left(\left[V_{1} \wedge \cdots \wedge V_{r-1}\right]\right\lrcorner B\right)= & \left(\left[D_{V_{r}}\left(V_{1}\right) \wedge \cdots \wedge V_{r-1}\right]+\ldots\right. \\
& \left.\left.+\left[V_{1} \wedge \cdots \wedge V_{r-2} \wedge D_{V_{r}}\left(V_{r-1}\right)\right]\right)\right\lrcorner B \\
& +\left(\left[V_{1} \wedge \cdots \wedge V_{r-1}\right]\right) \wedge D_{V_{r}} B .
\end{aligned}
$$

When the $V_{i}$ commute, we see that

$$
\int_{M^{k}}\left(V_{1} \wedge \cdots \wedge V_{r-1}\right)-D_{V_{r}}(B) d \mu=0 .
$$


Since $\left.\left.D_{V_{r}}(B)=d\left(V_{r}\right\lrcorner B\right)+V_{r}\right\lrcorner d B$ and $d\left(V_{r}-B\right)$ is bounding, it follows by our inductive hypothesis that

$$
\int_{M^{k}}\left(V_{p}^{v_{1}} \wedge \cdots \wedge V_{p}^{v_{n}}\right)-[(d B)(p)] d \mu(p)=0,
$$

which is what we had to prove.

We will now specialize to the case where $G=\mathbb{R}^{n}$ for some integer $n$. Since the pointwise ergodic theorem is known in this case, the known results about quasiregular points that hold when $G=\mathbb{R}$ also hold when $G=\mathbb{R}^{n}$. Thus, if $p \in M^{k}$ is such that for any continuous real-valued function $f$ on $M^{k}$,

$$
\lim _{a \rightarrow 0} \frac{1}{a^{n}} \int_{0}^{a} \cdots \int_{0}^{a} f\left[\left(t_{1} \ldots t_{n}\right) p\right] d t_{1} \wedge \cdots \wedge d t_{n}
$$

exists, we say that $p$ is quasi-regular. Then for any invariant probability measure $\mu$, the set $Q$ of all quasi-regular points has measure 1. Moreover, there exists for any quasi-regular point $p$ an invariant probability measure $\mu_{p}$ such that for each continuous function $f$,

$$
\int_{Q} f(q) d \mu_{p}(q)=\lim _{a \rightarrow 0} \frac{1}{a^{n}} \int_{0}^{a} \cdots \int_{0}^{a} f\left[\left(t_{1} \ldots t_{n}\right) p\right] d t_{1} \wedge \cdots \wedge d t_{n} .
$$

If we are given an $r$-vector $v$ over the Lie algebra of $\mathbb{R}^{n}$, we will denote by $A_{p}^{v}$ the element of $H_{r}\left(M^{k} ; \mathbb{R}\right)$ we have already associated with $\mu_{p}$ assuming $p$ is quasi-regular. For any closed $r$-form $B$, let $\bar{B}$ be the corresponding element of $H^{r}\left(M^{k} ; \mathbb{R}\right)$. If $p$ is quasi-regular, the value assigned to $\bar{B}$ by the homomorphism of $H^{r}\left(M^{k} ; \mathbb{R}\right)$ into $\mathbb{R}$ determined by $A_{p}^{v}$ is

$$
\left.\lim _{a \rightarrow \infty} \frac{1}{a^{n}} \int_{0}^{a} \cdots \int_{0}^{a}\left(V_{\left(t_{1} \ldots t_{n}\right) p}\right)-B_{\left(t_{1}, \ldots, t_{n}\right) p}\right) d t_{1} \wedge \cdots \wedge d t_{n},
$$

which we will denote by $A_{p}^{V} \cdot \bar{B}$

Hence, for any finite measure $\mu$ on $M^{k}$,

$$
\begin{aligned}
\int_{M^{k}} & A_{p}^{v} \cdot \bar{B} d \mu(p) \\
& =\int_{M^{k}}\left(\lim _{a \rightarrow \infty} \frac{1}{a^{n}} \int_{0}^{a} \cdots \int_{0}^{a}\left[\left(V_{\left(t_{1} \ldots t_{n}\right) p}-B_{\left(t_{1}, \ldots, t_{n}\right) p}\right) d t_{1} \wedge \cdots \wedge d t\right]\right) d \mu(p) \\
& =\lim _{a \rightarrow \infty} \int_{M^{k}}\left(\frac{1}{a^{n}} \int_{0}^{a} \cdots \int_{0}^{a}\left[\left(V_{\left(t_{1} \ldots t_{n}\right) p}-B_{\left(t_{1}, \ldots, t_{n}\right) p}\right) d t_{1} \wedge \cdots \wedge d t_{n}\right]\right) d \mu(p) .
\end{aligned}
$$

However, if $\mu$ is an invariant measure,

$$
\begin{aligned}
\int_{M^{k}}\left(V_{\left(t_{1} \ldots t_{n}\right) p}\right)-B_{\left(t_{1}, \ldots, t_{n}\right) p} d \mu(p) & \left.=\int_{M^{k}} V_{p}\right\lrcorner B_{p} d \mu(p) \\
& =A_{\mu}^{v} \cdot \bar{B}_{\mu}
\end{aligned}
$$

for any $\left(t_{1}, \ldots, t_{n}\right)$. It follows that $\int_{M^{k}} A_{p}^{v} \cdot \bar{B} d \mu(p)=A_{\mu}^{v} \cdot \bar{B}$. Thus we can conclude that

Theorem 2. If $\mu$ is a finite invariant measure for our action of $\mathbb{R}^{n}$ on $M^{k}$ and $v$ is any $r$-vector over the Lie algebra of $\mathbb{R}^{n}$, then

$$
\int_{M^{k}} A_{p}^{v} d \mu(p)=A_{\mu}^{v}
$$


Suppose that $G$ is compact and that $v$ is equal to $v_{1} \wedge \cdots \wedge v_{r}$, where the $v_{r}$ are commuting elements of the Lie algebra of $G$. There is a unique homomorphism of $\mathbb{R}^{r}$ into $G$ sending the standard basis for the Lie algebra of $\mathbb{R}^{r}$ into $\left(v_{1}, \ldots, v_{r}\right)$. Then our action of $G$ on $M^{k}$ determines an action of $\mathbb{R}^{r}$ on $M^{k}$.

Denote the closure of the image of this homomorphism by $H$. Then $H$ is a toroidal group; moreover, if $F$ is any continuous function on $M^{k}$ and $p \in M^{k}$,

$$
\lim _{a \rightarrow \infty} \frac{1}{a^{r}} \int_{0}^{a} \cdots \int_{0}^{a} F\left[\left(t_{1} \ldots, t_{r}\right) p\right] d t_{1} \wedge \cdots \wedge d t_{r}=\int_{H} F(h \cdot p) d m(h),
$$

where $m$ is the Haar measure on $H$. In particular, every point $p \in M^{k}$ is quasiregular under the action of $\mathbb{R}^{r}$, so that the asymptotic cycle $A_{p}^{v}$ arising from the $r$-vector $v$ is defined. If $B$ is any closed $r$-form on $M^{k}$ and $\bar{B}$ is the corresponding element of $H^{r}\left(M^{k}, \mathbb{R}\right)$, then the homomorphism of $H^{r}\left(M^{k}, \mathbb{R}\right)$ into $\mathbb{R}$ determined by $A_{p}^{v}$ sends $\bar{B}$ into

$$
\left.\int_{M^{k}} V_{q}^{v}\right\lrcorner B(q) d \mu_{p}(q)
$$

where $V^{v}$ is the $r$-vector field on $M^{k}$ determined by $v$. However, if $\Pi_{p}$ denotes the map of $H$ into $M^{k}$ sending $h \in H$ into $h \cdot p$ and $\Pi_{p}^{r}$ is the associated map sending $H^{r}\left(M^{k}, \mathbb{R}\right)$ into $H^{r}(H, \mathbb{R})$, then $\int_{M^{k}} V_{q}-B(q) d \mu_{p}(q)$, which equals

$$
\lim _{a \rightarrow \infty} \frac{1}{a^{r}} \int_{0}^{a} \cdots \int_{0}^{a}\left(V_{\left(t_{1} \ldots t_{r}\right) p}\right\lrcorner B_{\left(t_{1} \ldots t_{r}\right) p} d t_{1} \wedge \cdots \wedge d t_{r},
$$

is the image of $\Pi_{p}^{r}(\bar{B})$ under the homomorphism of $H^{r}(H, \mathbb{R})$ into $\mathbb{R}$ determined by $A_{e}^{v}$.

Here $e$ is the identity element of $H$. Our homomorphism of $\mathbb{R}^{r}$ into $H$ enables us to define an action of $\mathbb{R}^{r}$ on $H$, using the fact that $H$ acts on itself by translation. Since we may regard $v_{1}, \ldots, v_{r}$ as elements of the Lie algebra of $H$ and $e$ is quasiregular for our action of $\mathbb{R}^{r}$ on $H$, it makes sense to talk about the asymptotic cycle $A_{e}^{v}$ on $H$ that we get from $v=v_{1} \wedge \cdots \wedge v_{r}$.

The justification for our assertion lies in the fact that $\Pi_{p}$, which sends $e$ into $p$, is equivariant with respect to the actions of $\mathbb{R}^{r}$ on $H$ and $M^{k}$.

If $p_{1}$ and $p_{2}$ belong to $M^{k}$, then since $M^{k}$ is arcwise connected, $\Pi_{p_{1}}$ and $\Pi_{p_{2}}$ are homotopic maps. Consequently, $\Pi_{p_{1}}^{r}(\bar{B})=\Pi_{p_{2}}^{r}(\bar{B})$. Since $A_{e}^{v}$ is independent of $p$, it follows that $A_{p_{1}}^{v} \cdot \bar{B}=A_{p_{2}}^{v} \cdot \bar{B}$ for all $\bar{B}$. Therefore $A_{p_{1}}^{v}=A_{p_{2}}^{v}$ and consequently

Theorem 3. If $\mu_{1}$ and $\mu_{2}$ are invariant probability measures for the action of $G$ on $M^{k}$ and $v$ is as above, then $A_{\mu_{1}}^{v}=A_{\mu_{2}}^{v}$.

Proof. This follows from the fact that for any invariant measure $\mu, A_{\mu}^{v}=$ $\int_{M^{k}} A_{p}^{v} d \mu(p)$, while $A_{p}^{v}$ is the same for all points $p$.

\section{IV}

Given a flow arising from a vector field $V$ on a compact oriented manifold $M^{k}$ and an everywhere positive $k$-form $\Omega$ on $M^{k}$, the measure $\mu$ arising from $\Omega$ is invariant under the flow if and only if $V\lrcorner \Omega$ is closed. A theorem due to Arnold states that the asymptotic cycle $A_{\mu}^{V}$ arises by Poincaré duality from the closed form $V\lrcorner \Omega$. We wish to give the generalization of this that applies in our present context. 
Suppose that instead of the 1-vector field $V$, we have given $r$ commuting vector fields $V_{1}, \ldots, V_{r}$ on $M^{k}$. Suppose further that the measure associated with $\Omega$ is invariant under each of the flows arising from the vector fields $V_{i}$.

Lemma 1. $\left.\left(V_{1} \wedge \cdots \wedge V_{r}\right)\right\lrcorner \Omega$ is closed.

Proof. For any closed form $\alpha$ and vector field $W, D_{W} \alpha$ equals $\left.\left.W\right\lrcorner d \alpha+d(W\lrcorner \alpha\right)$. Therefore, if $\alpha$ is closed, $D_{W} \alpha=0$ if and only if $\left.W\right\lrcorner \alpha$ is closed. Since the operations $D_{V_{j}}$ and $D_{V_{i}}$ commute, for any $j$ between 1 and $r$,

$$
\left.0=D_{V_{j}}\left(V_{j-1}\right\lrcorner\left(\ldots\left(V_{2}\right\lrcorner\left(V_{1}\right\lrcorner \Omega\right) \ldots\right),
$$

so we see by induction that $\left.\left.V_{j}\right\lrcorner\left(V_{j-1}\right\lrcorner\left(\ldots\left(V_{2}\right\lrcorner\left(V_{1}\right\lrcorner \Omega\right) \ldots\right)$ is closed for each value of $j$. Thus $0=d\left(\left(V_{r} \wedge \cdots \wedge V_{1}\right)-\Omega\right)$.

Now we will prove

Theorem 4. The asymptotic cycle $A_{\mu}^{V}$ arising from the invariant measure $\mu$ and the r-vector field $V=V_{1} \wedge \cdots \wedge V_{r}$ can be obtained by Poincaré duality from the closed $(k-r)$-form $V-\Omega$.

Proof. If $B$ is any closed $r$-form on $M^{k}$, the homomorphism of $H^{r}\left(M^{k} ; \mathbb{R}\right)$ into $\mathbb{R}$ determined by $A_{\mu}$ sends $\bar{B}$ into $\int_{M^{k}}\left(\left(V_{1} \wedge \cdots \wedge V_{r}\right)-B\right) \Omega$. Our result then follows from the fact that

$$
\left.\left(\left(V_{1} \wedge \cdots \wedge V_{r}\right)-B\right) \Omega=B \wedge\left(\left[V_{1} \wedge \cdots \wedge V_{r}\right]\right\lrcorner \Omega\right) .
$$

\section{REFERENCES}

[1] Sol Schwartzman, Asymptotic Cycles, Annals of Mathematics, Vol. 66, 270-284, 1957. MR0088720(19:568i)

[2] Sol Schwartzman, Higher Dimensional Asymptotic Cycles, Canadian Journal of Mathematics, Vol. 55, 636-640, 2003. MR.1980617 (2004d:57036)

[3] Sol Schwartzman, Smooth Actions of $\mathbb{R}^{n}$, Proceedings of the A.M.S., Vol. 134, No. 2, 379-384, 2006. MR2176005 (2006j:57065)

[4] Sol Schwartzman, Asymptotic Cycles, Scholarpedia, 2008. Available online at http://www . scholarpedia.org/article/Asymptotic_cycles.

Department of Mathematics, University of Rhode Island, Kingston, Rhode Island 02881

E-mail address: solschwartzman@gmail.com 